\title{
The CUORE Bolometric Detector for Neutrinoless Double Beta Decay Searches
}

L. Cassina $^{10,11(\bowtie)}$, C. Alduino ${ }^{1}$, K. Alfonso ${ }^{2}$, D. R. Artusa ${ }^{1,3}$,

F. T. Avignone $\mathrm{III}^{1}$, O. Azzolini ${ }^{4}$, G. Bari ${ }^{5}$, F. Bellini $^{6,7}$, G. Benato ${ }^{8}$, A. Bersani ${ }^{9}$, M. Biassoni ${ }^{10,11}$, A. Branca ${ }^{12}$, C. Brofferio ${ }^{10,11}$, C. Bucci $^{3}$, A. Camacho ${ }^{4}$, A. Caminata ${ }^{9}$, L. Canonica ${ }^{3,13}$, X. G. Cao ${ }^{14}$, S. Capelli ${ }^{10,11}$, L. Cappelli ${ }^{3}$, L. Cardani $^{7}$, P. Carniti ${ }^{10,11}$, N. Casali ${ }^{7}$,

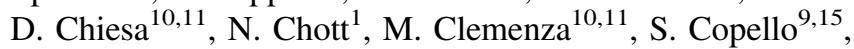

C. Cosmelli ${ }^{6,7}$, O. Cremonesi ${ }^{11}$, R. J. Creswick ${ }^{1}$, J. S. Cushman ${ }^{16}$, A. D’Addabbo ${ }^{3}$, D. D’Aguanno ${ }^{3,17}$, I. Dafinei ${ }^{7}$, C. J. Davis ${ }^{16}$,

S. Dell'Oro ${ }^{3,18}$, M. M. Deninno ${ }^{5}$, S. Di Domizio ${ }^{5,9}$,

M. L. Di Vacri ${ }^{3,19}$, A. Drobizhev ${ }^{8,20}$, D. Q. Fang ${ }^{14}$, M. Faverzani ${ }^{10,11}$, E. Ferri ${ }^{11}$, F. Ferroni ${ }^{6,7}$, E. Fiorini ${ }^{10,11}$, M. A. Franceschi ${ }^{21}$,

S. J. Freedman ${ }^{8,20}$, B. K. Fujikawa ${ }^{20}$, A. Giachero ${ }^{11}$, L. Gironi ${ }^{10,11}$,

A. Giuliani ${ }^{22}$, L. Gladstone ${ }^{13}$, P. Gorla ${ }^{3}$, C. Gotti ${ }^{10,11}$,

T. D. Gutierrez ${ }^{23}$, K. Han ${ }^{24}$, K. M. Heeger ${ }^{16}$,

R. Hennings-Yeomans ${ }^{8,20}$, H. Z. Huang ${ }^{2}$, G. Keppel ${ }^{4}$,

Y. G. Kolomensky ${ }^{8,20}$, A. Leder ${ }^{13}$, C. Ligi ${ }^{21}$, K. E. Lim ${ }^{16}$,

Y. G. $\mathrm{Ma}^{14}$, M. Maino ${ }^{10,11}$, L. Marini ${ }^{9,15}$, M. Martinez ${ }^{6,7,25}$,

R. H. Maruyama ${ }^{16}$, Y. Mei ${ }^{20}$, N. Moggi ${ }^{5,26}$, S. Morganti $^{7}$,

P. J. Mosteiro ${ }^{7}$, S. S. Nagorny ${ }^{3,18}$, T. Napolitano ${ }^{21}$, M. Nastasi ${ }^{10,11}$,

C. Nones ${ }^{27}$, E. B. Norman ${ }^{28,29}$, V. Novati ${ }^{22}$, A. Nucciotti ${ }^{10,11}$,

T. O'Donnell ${ }^{30}$, J. L. Ouellet ${ }^{13}$, C. E. Pagliarone ${ }^{3,17}$,

M. Pallavicini ${ }^{9,15}$, V. Palmieri ${ }^{4}$, L. Pattavina ${ }^{3}$, M. Pavan $^{10,11}$,

G. Pessina ${ }^{11}$, C. Pira ${ }^{4}$, S. Pirro ${ }^{3}$, S. Pozzi ${ }^{10,11}$, E. Previtali ${ }^{11}$,

C. Rosenfeld ${ }^{1}$, C. Rusconi ${ }^{1,3}$, M. Sakai ${ }^{2}$, S. Sangiorgio ${ }^{28}$,

D. Santone ${ }^{3,19}$, B. Schmidt ${ }^{20}$, J. Schmidt ${ }^{2}$, N. D. Scielzo ${ }^{28}$, V. $\operatorname{Singh}^{8}$,

M. Sisti ${ }^{10,11}$, L. Taffarello ${ }^{12}$, F. Terranova ${ }^{10,11}$, C. Tomei ${ }^{7}$,

M. Vignati ${ }^{7}$, S. L. Wagaarachchi ${ }^{8,20}$, B. S. Wang ${ }^{28,29}$, H. W. Wang ${ }^{14}$,

B. Welliver ${ }^{20}$, J. Wilson ${ }^{1}$, L. A. Winslow ${ }^{13}$, T. Wise ${ }^{16,31}$,

A. Woodcraft ${ }^{32}$, L. Zanotti ${ }^{10,11}$, G. Q. Zhang ${ }^{14}$, S. Zimmermann ${ }^{33}$, and S. Zucchelli ${ }^{5,26}$

1 Department of Physics and Astronomy,

University of South Carolina, Columbia, SC 29208, USA

2 Department of Physics and Astronomy, University of California,

Los Angeles, CA 90095, USA

3 INFN - Laboratori Nazionali del Gran Sasso, 67100 L'Aquila, Assergi, Italy

${ }^{4}$ INFN - Laboratori Nazionali di Legnaro, 35020 Padova, Legnaro, Italy

5 INFN - Sezione di Bologna, 40127 Bologna, Italy

${ }^{6}$ Dipartimento di Fisica, Sapienza Università di Roma, 00185 Rome, Italy

7 INFN - Sezione di Roma, 00185 Rome, Italy

8 Department of Physics, University of California, Berkeley, CA 94720, USA

${ }^{9}$ INFN - Sezione di Genova, 16146 Genoa, Italy 
10 Dipartimento di Fisica, Università di Milano-Bicocca, 20126 Milan, Italy

lorenzo.cassina@mib.infn.it

11 INFN - Sezione di Milano Bicocca, 20126 Milan, Italy

12 INFN - Sezione di Padova, 35131 Padua, Italy

13 Massachusetts Institute of Technology, Cambridge, MA 02139, USA

14 Shanghai Institute of Applied Physics, Chinese Academy of Sciences, Shanghai 201800, China

15 Dipartimento di Fisica, Università di Genova, 16146 Genoa, Italy

16 Department of Physics, Yale University, New Haven, CT 06520, USA

17 Dipartimento di Ingegneria Civile e Meccanica, Università degli Studi

di Cassino e del Lazio Meridionale, 03043 Cassino, Italy

18 INFN - Gran Sasso Science Institute, 67100 L'Aquila, Italy

19 Dipartimento di Scienze Fisiche e Chimiche,

Università dell'Aquila, 67100 L'Aquila, Italy

20 Nuclear Science Division, Lawrence Berkeley National Laboratory, Berkeley, CA 94720, USA

21 INFN - Laboratori Nazionali di Frascati, 00044 Frascati, Rome, Italy

22 CSNSM, Univ. Paris-Sud, CNRS/IN2P3,

Universit Paris-Saclay, 91405 Orsay, France

23 Physics Department, California Polytechnic State University, San Luis Obispo, CA 93407, USA

24 Department of Physics and Astronomy, Shanghai Jiao Tong University,

Shanghai 200240, China

25 Laboratorio de Fisica Nuclear y Astroparticulas,

Universidad de Zaragoza, 50009 Saragossa, Spain

${ }^{26}$ Dipartimento di Fisica e Astronomia,

Alma Mater Studiorum - Università di Bologna, 40127 Bologna, Italy

27 Service de Physique des Particules, CEA/Saclay, 91191 Gif-sur-Yvette, France

${ }^{28}$ Lawrence Livermore National Laboratory, Livermore, CA 94550, USA

29 Department of Nuclear Engineering,

University of California, Berkeley, CA 94720, USA

${ }^{30}$ Center for Neutrino Physics, Virginia Polytechnic Institute and State

University, Blacksburg, VA 24061, USA

31 Department of Physics, University of Wisconsin, Madison, WI 53706, USA

32 SUPA, Institute for Astronomy, University of Edinburgh, Blackford Hill, Edinburgh EH9 3HJ, UK

33 Engineering Division, Lawrence Berkeley National Laboratory, Berkeley, CA 94720, USA

\begin{abstract}
The Cryogenic Underground Observatory for Rare Events (CUORE) is the first bolometric experiment reaching the 1-ton scale. The detector consists of an array of $988 \mathrm{TeO}_{2}$ crystals arranged in 19 towers. The construction of the experiment and the installation of the detector was completed in August 2016. In this paper, the technical challenges of the construction, the design choices and measured performance of the electronic instrumentation are presented.
\end{abstract}




\section{Introduction}

Although in the Standard Model neutrinos are classified as Dirac massless particles, the discovery of neutrino oscillations [1] revealed that neutrinos are massive thereby provided the first evidence of new physics. Nevertheless, oscillations do not give information about the absolute mass scale of the neutrino nor about the hierarchy of its different mass states. Moreover, there is no evidence whether neutrinos are Dirac or Majorana particles, i.e. if they are their own antiparticles. Neutrinoless double-beta decay $(0 v \beta \beta)$ has the unique potential to provide insight into the above issues. This extremely rare $\left(T_{1 / 2}^{0 v} \sim 10^{25}-10^{26} \mathrm{yr}\right)$ lepton-number-violating process consists of two simultaneous beta decay in which the two neutrinos annihilates, so that only the two electrons are emitted from the nuclide. Unlike the double beta decay, the energy spectrum of two electrons produced in the $0 v \beta \beta$ results in a narrow peak at the Q-value, whose resolution is only limited by the detection performance. Experimental evidence the $0 v \beta \beta$ would provide information about the neutrino mass hierarchy and absolute mass scale from decay half-life.

One of the most important figure of merit of such experiments is the sensitivity $\left(S^{O v}\right)$, defined as the life-time corresponding to the minimum detectable number of events over the background at a given confidence level. In the approximation of nonzero background, $S^{0 v}$ increases proportionally to the isotopic abundance of the radionuclide of interest, to the square root of the detector mass and experiment lifetime, and inversely proportional to the square root of the energy resolution at the Qvalue and the background index, i.e. the number of spurious events per unit of energy, time and mass at the Q-value. In order to push the sensitivity up to $10^{25}-10^{26} \mathrm{yr}$, very large masses must be observed for several years and excellent energy resolution and extremely low background must be achieved. The Cryogenic Underground Observatory for Rare Events, CUORE [2], is the first ton-scale experiment that searches the $0 v \beta \beta$ in ${ }^{130} \mathrm{Te}$. In this paper, an overlook on the design and status of CUORE is described, including the detector assembly and the cryogenics commissioning. A brief overview on the electronic system is also provided in Sect. 3.

\section{The CUORE Experiment}

CUORE is a bolometric experiment located in the Hall A of the underground Laboratori Nazionali del Gran Sasso (LNGS), Italy, which offers a covering of $\sim 3700$ m.w.e. (meter water equivalent) above the experimental area. The CUORE detector consists of an array of $988 \mathrm{TeO}_{2}$ bolometers arranged in a compact cylindrical configuration of 19 towers, each made from the assembly of 4 columns of 13 crystals each, so that a single tower hosts 52 cubic bolometers, $5 \times 5 \times 5 \mathrm{~cm}^{3}$ each. The base blocks composing a bolometer detector and are shown in Fig. 1. Each crystal is equipped with a Neutron Transmutation Doping (NTD) thermistor, a sensor whose resistivity exponentially depends on the operating temperature. A heating resistor is also thermally coupled to the bolometer to provide a reference signal for the detector response stabilization. Bolometers are particularly suitable for the search of $0 v \beta \beta$ decay as they ensure an 


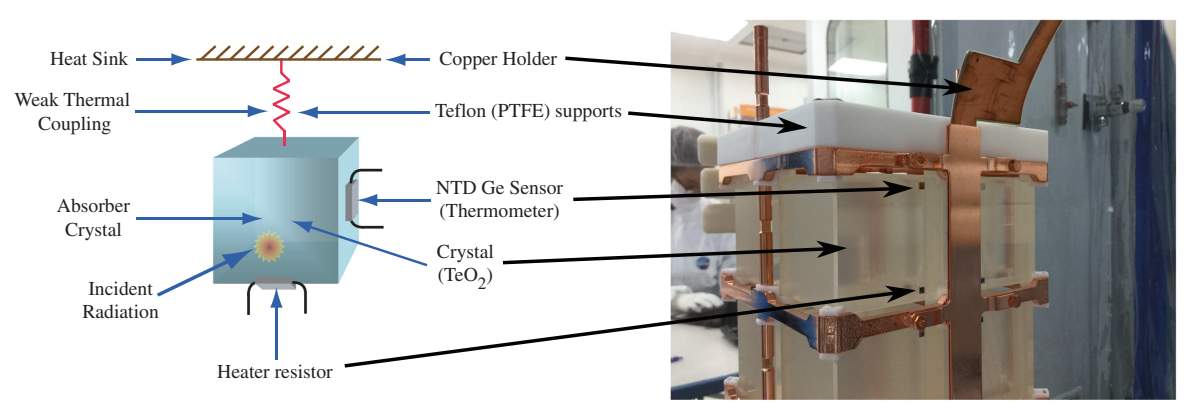

Fig. 1. Simplified scheme and a picture of a bolometer CUORE detector. The sensitive crystal is weakly coupled to the thermal bath through an insulating Teflon frame. The NTD thermistor and the heater resistor are also pointed out.

excellent energy resolution, no dead layers, wide choice of materials and the potential to compose ton scale detectors.

CUORE uses $\mathrm{TeO}_{2}$ cubic bolometer of $750 \mathrm{~g}$ each, with a thermal capacitance of $\sim 2 \mathrm{~nJ} / \mathrm{K}$ at $\sim 10 \mathrm{mk}$. The excellent performance of such crystals was already demonstrated by the pilot experiment CUORICINO [3], which also represented a fundamental phase to refine all the stages for the CUORE tower construction. The crystals were placed in a clean room where a custom-made robotic arm glued both the thermistor and the heater on the crystals. The towers were assembled in a glow box where the copper structure, the teflon supports of the crystals and the flat cable for the electrical system were mounted. Finally all the sensors and heaters were bounded to the electrical pads. After the assembly, the towers were kept inside boxes fluxed with nitrogen. The towers were finally installed inside the cryostat during August 2016, in a controlled radon-free atmosphere. All these steps allow to achieve an extremely high radiopurity level (background goal: $B \sim 10^{-2} \mathrm{keV}^{-1} \mathrm{~kg}^{-1} \mathrm{yr}^{-1}$ ). The $\sim 1$ ton detector was cooled down at the baseline temperature of $\sim 10 \mathrm{mk}$ in January 2017 . In order to achieve such low temperature and long term stability, a custom dilution refrigerator was designed and commissioned in March 2016. The cryostat is mechanically decoupled from the detector by means of a multi-stage suspension system in order to avoid that frictions could inject thermal power. The target energy resolution of CUORE is $\sigma_{\mathrm{E}} / \mathrm{E}<10^{-3}$, which corresponds to $5 \mathrm{keV} \mathrm{FWHM}$ at $\mathrm{Q}_{\mathrm{Te}}=2528 \mathrm{keV}$.

\section{The Electronic System}

The main tasks the electronic system must fulfill is the detector bias, the signal readout, its amplification and the stabilization of the detector response. As the detector is supposed to run for several years, an extremely high stability is needed. Furthermore, the electronic noise must be minimized as it spoils the achievable energy resolution. The power supply for the electronic system and the stable and low noise reference for the detector bias is provided by a three stage chain: a Lambda programmable AD/DC converters, from TDK, feeds a custom designed, low noise DC/DC converter. To filter 
the residual ripple noise, a linear power supply has been developed to provide the frontend bias [4]. This equipment is located at the top of the cryostat, inside a Skudotech Faraday cage, and connected to the low temperature crystals by a twisted pair cable. By using such architecture, most of the low frequency microphonic noise results in a common mode signal among the twisted pair and it can be rejected by the fully differential, low noise, front-end system [5]. The signal is acquired by a voltage sensitive pre-amplifier feeding an adjustable gain amplifier. The signal is finally filtered by a Bessel stage that optimizes the signal-to-noise ratio and behaves as anti-aliasing filter before the digitization. The full system is remotely controlled and managed by an optically decoupled slow monitoring system. The whole equipment was installed during 2016 and the optimization of the electronic operational parameters and the detector working point was completed in late 2016. Figure 2 shows one of the first pulses acquired by the CUORE experiment in January 2017.

Temperature fluctuations usually occur in cryogenic experiment and affect the response of bolometers and thermistors. The electronic system is equipped with an apparatus able to provide a very stable voltage step across the heaters, so that a thermal pulse can be injected to the bolometers. Such pulses are used as reference signal to compensate for the gain drifts. The pulser boards have been designed to achieve an energy resolution of $\sim 2 \mathrm{ppm}$ at $1 \mathrm{MeV}$ and each board was calibrated to ensure a thermal drift of $\sim 0.1 \mathrm{ppm} /{ }^{\circ} \mathrm{C}$ in a operating temperature range of $20-60{ }^{\circ} \mathrm{C}$. The efficacy of the described calibrating system is shown in Fig. 3. Data acquired in two different datasets are added to compose the energy spectrum. After the stabilization, the gain fluctuations between the runs are completely compensated and the energy peak can be reconstructed with the expected resolution.

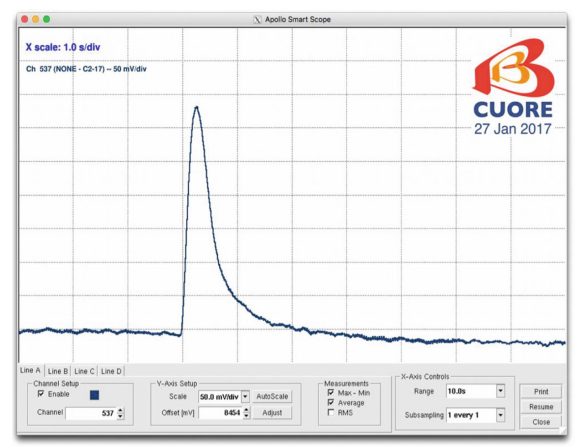

Fig. 2. First pulse acquired by the fully assembled detector of the CUORE experiment (January 2017).

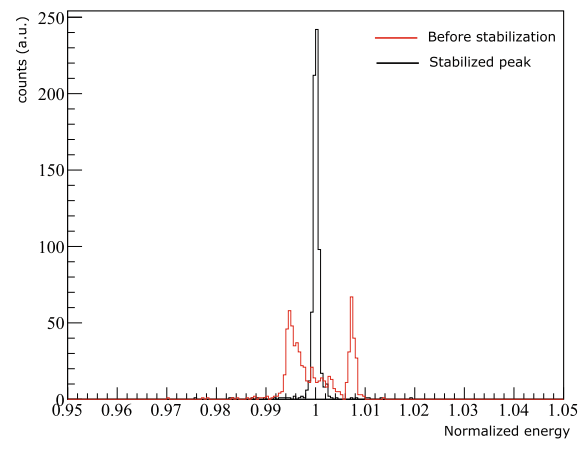

Fig. 3. Pulser peak before and after the detector response stabilization, acquired during the cryostat commissioning test (August 2016). 


\section{Conclusions}

This paper presented the status of CUORE, a ton scale experiment researching the neutrinoless double beta decay in ${ }^{130} \mathrm{Te}$ adopting a bolometric technique. The detector installation has been completed successfully, the CUORE cryostat has been commissioned and reached the baseline temperature in January 2017. The electronic system was installed in first half of 2016 and then optimized on-site. The detector working point and setup optimization is going to be completed and the data taking is now commencing.

\section{References}

1. Bellini, G., et al.: Neutrino oscillations. In: Advances in High Energy Physics (2014)

2. Arnaboldi, C., et al.: CUORE: a cryogenic underground observatory for rare events. Nucl. Instrum. Methods Phys. Res. Sect. A 518(3), 775 (2004)

3. Arnaboldi, C., et al: Results from a search for the $0 v \beta \beta$-decay of ${ }^{130}$ Te. Phys. Rev. C 78, 035502 (2008)

4. Carniti, P., et al.: Very low noise AC/DC power supply systems for large detector arrays. Rev. Sci. Instrum. 86, 124703 (2015)

5. Arnaboldi, C., et al.: The design of the input stage for the very front-end of the CUORE experiment. Low Temp. Phys. 151, 964-970 (2008) 